\title{
Relativistic continuum-continuum coupling in the dissociation of halo nuclei
}

\author{
C. A. Bertulani ${ }^{1, \text { * }}$ \\ ${ }^{1}$ Department of Physics, University of Arizona, Tucson, Arizona 85721
}

(Dated: July 5, 2018)

\begin{abstract}
A relativistic coupled-channels theory for the calculation of dissociation cross sections of halo nuclei is developed. A comparison with non-relativistic models is done for the dissociation of ${ }^{8} \mathrm{~B}$ projectiles. It is shown that neglecting relativistic effects leads to seizable inaccuracies in the extraction of the astrophysical S-factor for the proton+beryllium radiative capture reaction.

PACS numbers: 25.60.+v;25.70.De;25.70.Mn
\end{abstract}

Reactions with radioactive nuclear beams have quickly become a major research area in nuclear physics. Among the newly developed techniques, the Coulomb dissociation method is an important tool to obtain electromagnetic matrix elements between continuum and bound states of rare nuclear isotopes [1]. These matrix elements play an essential role in nuclear astrophysics. At low continuum energies, they are the same as the ones involved in radiative capture processes of astrophysical interest. In particular, the Coulomb dissociation of ${ }^{8} \mathrm{~B}$ projectiles allows to extract information on the radiative capture reaction $\mathrm{p}+{ }^{7} \mathrm{Be} \rightarrow{ }^{8} \mathrm{~B}+\gamma$, of relevance for the standard solar model and the production of high-energy neutrinos in the sun 2].

The dissociation of weakly-bound nuclei, or halo nuclei, is dominated by the Coulomb interaction, although the nuclear interaction with the target cannot be neglected in most cases. The final state interaction of the fragments with the target leads to important continuum-continuum and continuum-bound-state couplings which appreciably modify the reaction dynamics. Higher-order couplings are more relevant in the dissociation of halo nuclei due to their low binding. A known example is the "postacceleration" (or "reacceleration") effect observed in the dissociation of ${ }^{11} \mathrm{Li}$ projectiles [3, 4, 5, 6] .

Two methods have been devised to study higher-order effects in projectile dissociation. The method introduced in ref. [6] uses the direct solution of the Schrödinger equation (DSSE) by space-time discretization. One starts with a ground-state wavefunction, propagates it through each time-step, and after sufficiently long time the iterated wavefunction is projected into a specific channel of interest. Another method discretizes the continuum wavefunctions $|c\rangle$ and uses them as input to calculate the matrix elements $\left\langle c^{\prime}\left|V_{\text {int }}\right| c\right\rangle$ and $\left\langle c\left|V_{\text {int }}\right| b\right\rangle$, where $|b\rangle$ denotes a bound state [3]. The matrix elements are then used in a coupled-channels calculation for transition amplitudes to dissociation channels. This is known as continuum discretized coupled-channels (CDCC) method and was introduced by Rawitscher [7] to study nuclear breakup reactions of the type $a+A \rightarrow b+c+A$. It has

*Electronic address: bertulani@physics.arizona.edu been used extensively in the study of breakup reactions with stable [8] and unstable nuclear projectiles [3, ㅁ, 10]

Special relativity, an obviously important concept in physics, is quite often neglected in the afore mentioned dynamical calculations. Most rare isotope facilities use projectile dissociation at $100 \mathrm{MeV} /$ nucleon. At these energies, relativistic effects are expected to be of the order of $10 \%$. Relativistic effects are accounted for in the collision kinematics, in first-order perturbation calculations, but not in dynamical calculations used sofar in the analysis of experiments. They also enter the dynamics in a non-linear, often unpredictable, way. The reason why these effects have not been considered before is due to the inherent theoretical difficulty in defining a nuclear potential between many-body relativistic systems. Due to retardation, an attempt to use a microscopic description starting from binary collisions of the constituents is not possible. A successful approach, known as "Dirac phenomenology", has been achieved for nucleon-nucleus scattering [1]. But for nucleus-nucleus collisions a reasonable account of these features has not yet been accomplished. In the present letter, the case of dissociation of ${ }^{8} \mathrm{~B}$ projectiles is studied. The major contribution comes from the Coulomb interaction with well-known relativistic transformation properties. A coupled-channels method based on the eikonal approximation with relativistic ingredients is developed and compared to semiclassical methods 6]. Here I omit the consideration of other corrections in the eikonal treatment of the scattering involving halo nuclei which have also been shown to play a relevant role (see, e.g., refs. [12, 13]).

Let us consider the Klein-Gordon (KG) equation with a potential $V_{0}$ which transforms as the time-like component of a four-vector [1]. For a system with total energy $E$ (including the rest mass $M$ ), the $\mathrm{KG}$ equation can be cast into the form of a Schrödinger equation (with $\hbar=c=1),\left(\nabla^{2}+k^{2}-U\right) \Psi=0$, where $k^{2}=\left(E^{2}-M^{2}\right)$ and $U=V_{0}\left(2 E-V_{0}\right)$. When $V_{0} \ll M$, and $E \simeq M$, one gets $U=2 M V_{0}$, as in the nonrelativistic case. The condition $V_{0} \ll M$ is met in peripheral collisions between nuclei at all collision energies. Thus, one can always write $U=2 E V_{0}$. A further simplification is to assume that the center of mass motion of the incoming projectile and outgoing fragments is only weakly modulated by the potential $V_{0}$. To get the dy- 
namical equations, one discretizes the wavefunction in terms of the longitudinal center-of-mass momentum $k_{z}$, using the ansatz

$$
\Psi=\sum_{\alpha} \mathcal{S}_{\alpha}(z, \mathbf{b}) \exp \left(i k_{\alpha} z\right) \phi_{k_{\alpha}}(\boldsymbol{\xi})
$$

In this equation, $(z, \mathbf{b})$ is the projectile's center-of-mass coordinate, with $\mathbf{b}$ equal to the transverse coordinate. $\phi(\boldsymbol{\xi})$ is the projectile intrinsic wavefunction and $(k, \mathbf{K})$ is the projectile's center-of mass momentum with longitudinal momentum $k$ and transverse momentum $\mathbf{K}$. There are hidden, uncomfortable, assumptions in eq. 1] The separation between the center of mass and intrinsic coordinates is not permissible under strict relativistic treatments. For high energy collisions we can at best justify eq. 1 for the scattering of light projectiles on heavy targets. Eq. 11 is only reasonable if the projectile and target closely maintain their integrity during the collision, as in the case of very peripheral collisions.

Neglecting the internal structure means $\phi_{k_{\alpha}}(\boldsymbol{\xi})=1$ and the sum in eq. 1 1 reduces to a single term with $\alpha=0$, the projectile remaining in its ground-state. It is straightforward to show that inserting eq. 11 in the KG equation $\left(\nabla^{2}+k^{2}-2 E V_{0}\right) \Psi=0$, and neglecting $\nabla^{2} \mathcal{S}_{0}(z, \mathbf{b})$ relative to $i k \partial_{Z} \mathcal{S}_{0}(z, \mathbf{b})$, one gets $i k \partial_{Z} \mathcal{S}_{0}(z, \mathbf{b})=$ $E V_{0} \mathcal{S}_{0}(z, \mathbf{b})$, which leads to the center of mass scattering solution $\mathcal{S}_{0}(z, \mathbf{b})=\exp \left[-i v^{-1} \int_{-\infty}^{z} d z^{\prime} V_{0}\left(z^{\prime}, \mathbf{b}\right)\right]$, with $v=k / E$. Using this result in the LippmannSchwinger equation, one gets the familiar result for the eikonal elastic scattering amplitude, i.e. $f_{0}=$ $-i(k / 2 \pi) \int d \mathbf{b} \exp (i \mathbf{Q} \cdot \mathbf{b})\{\exp [i \chi(\mathbf{b})]-1\}$, where the eikonal phase is given by $\exp [i \chi(\mathbf{b})]=\mathcal{S}_{0}(\infty, \mathbf{b})$, and $\mathbf{Q}=\mathbf{K}^{\prime}-\mathbf{K}$ is the transverse momentum transfer. Therefore, the elastic scattering amplitude in the eikonal approximation has the same form as that derived from the Schrödinger equation in the non-relativistic case.

For inelastic collisions we insert eq. 1 in the KG equation and use the orthogonality of the intrinsic wavefunctions $\phi_{k_{\alpha}}(\boldsymbol{\xi})$. This leads to a set of coupled-channels equations for $\mathcal{S}_{\alpha}$ :

$$
\left(\nabla^{2}+k^{2}\right) \mathcal{S}_{\alpha} \mathrm{e}^{i k_{\alpha} z}=\sum_{\alpha}\left\langle\alpha|U| \alpha^{\prime}\right\rangle \mathcal{S}_{\alpha^{\prime}} \mathrm{e}^{i k_{\alpha^{\prime}} z},
$$

with the notation $|\alpha\rangle=\left|\phi_{k_{\alpha}}\right\rangle$. Neglecting terms of the

form $\nabla^{2} \mathcal{S}_{\alpha}(z, \mathbf{b})$ relative to $i k \partial_{Z} \mathcal{S}_{\alpha}(z, \mathbf{b})$, eq. 2 reduces to

$$
i v \frac{\partial \mathcal{S}_{\alpha}(z, \mathbf{b})}{\partial z}=\sum_{\alpha^{\prime}}\left\langle\alpha\left|V_{0}\right| \alpha^{\prime}\right\rangle \mathcal{S}_{\alpha^{\prime}}(z, \mathbf{b}) \mathrm{e}^{i\left(k_{\alpha^{\prime}}-k_{\alpha}\right) z} .
$$

The scattering amplitude for the transition $0 \rightarrow \alpha$ is given by

$$
f_{\alpha}(\mathbf{Q})=-\frac{i k}{2 \pi} \int d \mathbf{b} \exp (i \mathbf{Q} \cdot \mathbf{b})\left[S_{\alpha}(\mathbf{b})-\delta_{\alpha, 0}\right]
$$

with $S_{\alpha}(\mathbf{b})=\mathcal{S}_{\alpha}(z=\infty, \mathbf{b})$. The set of equations 3 and 4 are the relativistic-CDCC equations (RCDCC).

I have used the RCDCC equations to study the dissociation of ${ }^{8} \mathrm{~B}$ projectiles at high energies. The energies transferred to the projectile are small, so that the wavefunctions can be treated non-relativistically in the projectile frame of reference. In this frame the wavefunctions will be described in spherical coordinates, i.e. $|\alpha\rangle=|j l J M\rangle$, where $j, l, J$ and $M$ denote the angular momentum numbers characterizing the projectile state. Eq. 3 is Lorentz invariant if the potential $V_{0}$ transforms as the time-like component of a four-vector. The matrix element $\left\langle\alpha\left|V_{0}\right| \alpha^{\prime}\right\rangle$ is also Lorentz invariant, and we can therefore calculate them in the projectile frame.

The longitudinal wavenumber $k_{\alpha} \simeq\left(E^{2}-M^{2}\right)^{1 / 2}$ also defines how much energy is gone into projectile excitation, since for small energy and momentum transfers $k_{\alpha}^{\prime}-k_{\alpha}=\left(E_{\alpha}^{\prime}-E_{\alpha}\right) / v$. In this limit, eqs. 3] and [4 reduce to semiclassical coupled-channels equations, if one uses $z=v t$ for a projectile moving along a straightline classical trajectory, and changing to the notation $\mathcal{S}_{\alpha}(z, b)=a_{\alpha}(t, b)$, where $a_{\alpha}(t, b)$ is the time-dependent excitation amplitude for a collision wit impact parameter $b$ (see eqs. 41 and 76 of ref. [14]). Here I use the full version of eq. 4

If the state $|\alpha\rangle$ is in the continuum (positive proton $+{ }^{7} \mathrm{Be}$ energy) the wavefunction is discretized according to $\left|\alpha ; E_{\alpha}\right\rangle=\int d E_{\alpha}^{\prime} \Gamma\left(E_{\alpha}^{\prime}\right)\left|\alpha ; E_{\alpha}^{\prime}\right\rangle$, where the functions $\Gamma\left(E_{\alpha}\right)$ are assumed to be strongly peaked around the energy $E_{\alpha}$ with width $\Delta E$. For convenience the histogram set (eq. 3.6 of ref. [3]) is chosen. The inelastic cross section is obtained by solving the RCDCC equations and using $d \sigma / d \Omega d E_{\alpha}=\left|f_{\alpha}(\mathbf{Q})\right|^{2} \Gamma^{2}\left(E_{\alpha}\right)$.

The potential $V_{0}$ contains contributions from the nuclear and the Coulomb interaction. The nuclear potentials are constructed along traditional lines of nonrelativistic theory. The standard double-folding approximation $V_{N}^{(a T)}(\mathbf{R})=\int \rho_{a}(\mathbf{r}) v_{0}(\mathbf{s}) \rho_{T}\left(\mathbf{r}^{\prime}\right)$ is used, where $v_{0}(\mathbf{s})$ is the effective nucleon-nucleon interaction, with $\mathbf{s}=\mathbf{r}+\mathbf{R}-\mathbf{r}^{\prime}$. The ground-state densities for the proton, ${ }^{7} \mathrm{Be}\left(\rho_{a}\right)$, and $\mathrm{Pb}$ target $\left(\rho_{T}\right)$, are taken from ref. [15]. The M3Y effective interaction [16] is used for $v_{0}(\mathbf{s})$. The nucleus-nucleus potential is expanded into $l=0,1,2$ multipolarities. These potentials are then transformed as the time-like component of a four-vector, as described above (see also ref. 14]). The multipole expansion of the Coulomb interaction in the projectile frame including retardation and assuming a straight-line motion has been derived in ref. [14]. The first term (monopole) of the expansion is the retarded LienardWiechert potential which does not contribute to the excitation, but to the center of mass scattering. Due to its long range, it is hopeless to solve eq. 3 with the Coulomb monopole potential, as $\mathcal{S}_{\alpha}(z, \mathbf{b})$ will always diverge. This can be rectified by using the regularization scheme $S_{\alpha}(\mathbf{b}) \rightarrow \exp \left[i\left(2 \eta \ln (k b)+\chi_{a}(b)\right)\right] S_{\alpha}(\mathbf{b})$, where $\eta=Z_{P} Z_{T} / \hbar v$ and the $S_{\alpha}(\mathbf{b})$ on the right-hand side is now calculated without inclusion of the Coulomb 


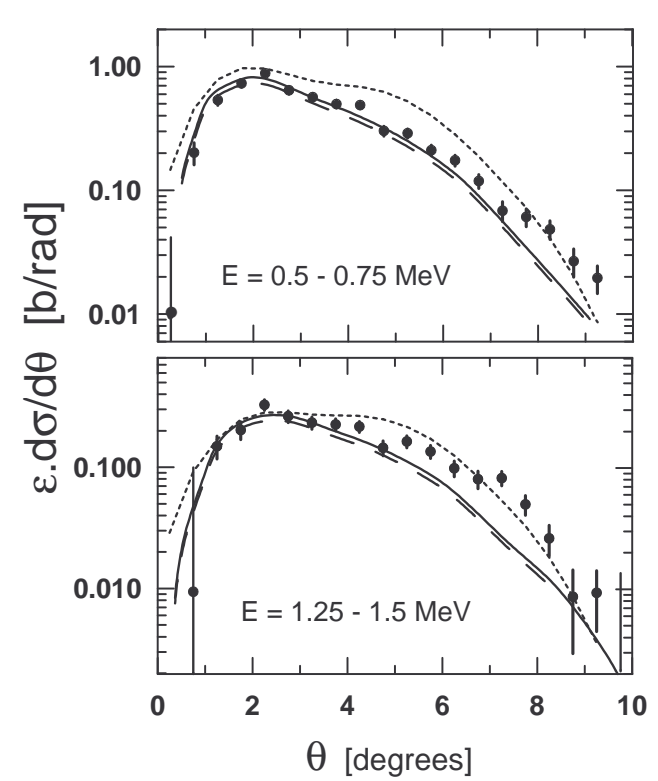

FIG. 1: Angular distributions for the dissociation reaction ${ }^{8} \mathrm{~B}+\mathrm{Pb} \rightarrow \mathrm{p}+{ }^{7} \mathrm{Be}+\mathrm{Pb}$ at $50 \mathrm{MeV} /$ nucleon. Data are from ref. 24] and are corrected for the detection efficiency $\varepsilon$. The dotted curve is the first-order perturbation result of ref. [25]. The solid curve is the RCDCC calculation. The dashed curve is obtained with the replacement of $\gamma$ by unity in the nuclear and Coulomb potentials.

monopole potential in eq. 3 The purely imaginary absorption phase, $\chi_{a}(b)$, was introduced to account for absorption at small impact parameters. It has been calculated using the imaginary part of the "t $\rho \rho$ " interaction [17], with the ${ }^{8} \mathrm{~B}$ density calculated with a modified Hartree-Fock model [18]. The E1 and E2 interactions, taken from ref. [14] replacing $v t \rightarrow z$. Explicitly,

$V_{E 1 \mu}=\sqrt{\frac{2 \pi}{3}} \xi Y_{1 \mu}(\hat{\boldsymbol{\xi}}) \frac{\gamma Z_{T} e_{1} e}{\left(b^{2}+\gamma^{2} z^{2}\right)^{3 / 2}}\left\{\begin{array}{ccc}\mp b & \text { if } & \mu= \pm 1 \\ \sqrt{2} z & \text { if } & \mu=0,\end{array}\right.$

for the E1 (electric dipole) field, and

$$
\begin{aligned}
V_{E 2 \mu} & =\sqrt{\frac{3 \pi}{10}} \xi^{2} Y_{2 \mu}(\hat{\boldsymbol{\xi}}) \frac{\gamma Z_{T} e_{2} e}{\left(b^{2}+\gamma^{2} z^{2}\right)^{5 / 2}} \\
& \times\left\{\begin{array}{c}
b^{2} \text { if } \mu= \pm 2 \\
\mp 2 \gamma^{2} b z \quad \text { if } \mu= \pm 1 \\
\sqrt{2 / 3}\left(2 \gamma^{2} z^{2}-b^{2}\right) \text { if } \mu=0 .
\end{array}\right.
\end{aligned}
$$

for the E2 (electric quadrupole) field, where $e_{1}=\frac{3}{8} e$ and $e_{2}=\frac{53}{64} e$ are effective charges for $p+{ }^{7}$ Be. For $\gamma \rightarrow 1$ these potentials reduce to the non-relativistic multipole fields (see, e.g., eq. 2 of ref. [19]) in distant collisions.

A single-particle model was used for ${ }^{8} \mathrm{~B}$ with a WoodsSaxon potential adjusted to reproduce the binding energy of $0.139 \mathrm{MeV}$ 20, 21, 22]. I follow the method of ref. [3] and divide the continuum into bins of widths $\Delta E_{\alpha}=100$ $\mathrm{keV}$, centered at $E_{\alpha}=0.01,0.11,0.21, \ldots, 1.01 \mathrm{MeV}$, bins

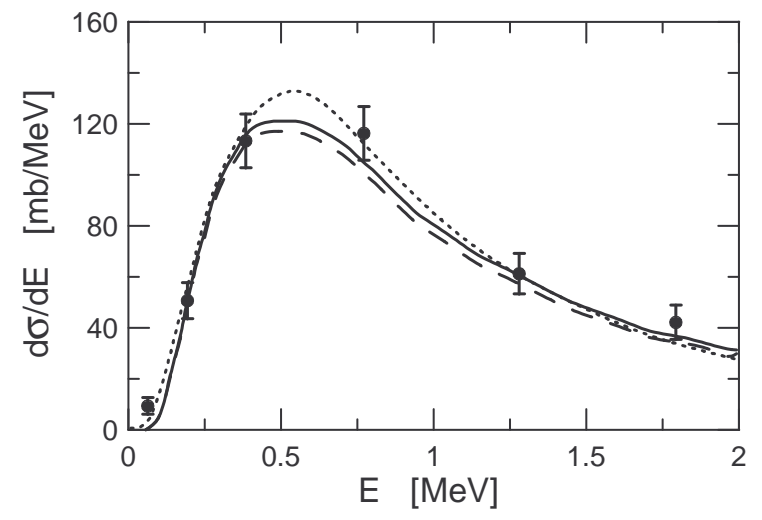

FIG. 2: Cross sections for the dissociation reaction ${ }^{8} \mathrm{~B}+\mathrm{Pb} \rightarrow$ $\mathrm{p}+{ }^{7} \mathrm{Be}+\mathrm{Pb}$ at $83 \mathrm{MeV} /$ nucleon and for $\theta_{8}<1.8^{0}$. Data are from ref. 23]. The dotted curve is the first-order perturbation result. The solid curve is the RCDCC calculation. The dashed curve is obtained with the replacement of $\gamma$ by unity in the nuclear and Coulomb potentials.

of widths $\Delta E_{\alpha}=250 \mathrm{keV}$, centered at $E_{\alpha}=1.25,1.5 \ldots$, $2.0 \mathrm{MeV}$, and bins of width $\Delta E_{\alpha}=0.75 \mathrm{MeV}$, centered at $E_{\alpha}=2.50,3.25, \ldots, 10.00 \mathrm{MeV}$. Each state $\alpha$ is a combination of energy and angular momentum quantum numbers $\alpha=\left\{E_{\alpha}, l, j, J, M\right\}$. Continuum $s, p, d$ and $f$ waves in ${ }^{8} \mathrm{~B}$ were included.

The calculations with the RCDCC equations were compared to the data of refs. 24] and 23]. The results were folded with the efficiency matrix as well as the energy averaging procedures explained in ref. 24] and provided by the RIKEN collaboration [24]. At $83 \mathrm{MeV} /$ nucleon, the angular distribution was chosen to match the same scattering angles referred to in ref. 23.

Figure 11 shows the angular distributions for the dissociation reaction ${ }^{8} \mathrm{~B}+\mathrm{Pb} \rightarrow \mathrm{p}+{ }^{7} \mathrm{Be}+\mathrm{Pb}$ at 50 $\mathrm{MeV} /$ nucleon. Data are from ref. 24]. The relative energy $E$ between the proton and ${ }^{7} \mathrm{Be}$ is averaged over the energy intervals $\mathrm{E}=0.5-0.7 \mathrm{MeV}$ (upper panel) and $E=1.25-1.5 \mathrm{MeV}$ (lower panel). The dotted curve is the first-order perturbation result reported in ref. 25]. The solid curve is the result of the RCDCC calculation. The dashed curve is obtained with the replacement of $\gamma$ (Lorentz factor) by unity in the nuclear and Coulomb potentials. The dashed curve is on average 3-6\% lower than the solid curves in figure 1 . Using non-relativistic potentials yields results always smaller than the full RCDCC calculation. It is a non-trivial task to predict what the relativistic corrections do in a coupled-channels calculation.

Figure 2 shows the relative energy spectrum between the proton and the ${ }^{7} \mathrm{Be}$ after the breakup of ${ }^{8} \mathrm{~B}$ on lead targets at $83 \mathrm{MeV} /$ nucleon. The data are from ref. [23]. In this case, the calculation was restricted to $b>30 \mathrm{fm}$. The dotted curve is the first-order perturbation calculation, the solid curve is the RCDCC calculation, and the dashed curve is obtained with the replacement of $\gamma$ 
by unity in the nuclear and Coulomb potentials. The difference between the solid and the dashed-curve is of the order of $4-9 \%$. I have also used the DSSE method, described in ref. [6], to compute the same spectrum, with the same partial waves, assuming a large lower cutoff in the parameter of $b=30 \mathrm{fm}$. This would justify a semiclassical limit. The same relativistic nuclear and Coulomb potentials have been used in both calculations. The difference between the two results (the RCDCC and the DSSE) is very small (less than $2 \%$ ) for the whole range of the spectrum. To my knowledge such a comparison has never been made before. This is a proof that the two methods yield the same result, if the same potentials are used, and as long as large b's are considered. Such a comparison is only possible because the same potential was used for the bound-state and the continuum. The DSSE method [6] does not allow for use of different potentials for $\mathrm{p}+{ }^{7} \mathrm{~B}$. This is not a problem in the RCDCC method, since the states $|b\rangle$ and $|c\rangle$ can be calculated within any level of sophistication, beyond the simple potential model adopted here. In this respect, the RCDCC is superior than the DSSE method and is more suitable for an accurate description of dynamical calculations.

The conclusions drawn in this work are crucial in the analysis of Coulomb breakup experiments at high bombarding energies, as the GSI experiment at 254 $\mathrm{MeV} /$ nucleon 26]. In table $1 \mathrm{I}$ show the calculations for the correction factor $\Delta=\left(\sigma^{R C D C C}-\sigma^{C D C C}\right) / \sigma^{C D C C}$ for the dissociation of ${ }^{8} \mathrm{~B}$ on lead targets at 3 bombarding energies. $E$ is the relative energy of the proton and ${ }^{7} \mathrm{Be}$. One sees that the relativistic corrections tend to in- crease the cross sections. At $250 \mathrm{MeV} /$ nucleon they can reach a $15 \%$ value. This has been treated before in firstorder perturbation theory, but not in the dynamical calculations with continuum-continuum coupling used in the experimental analysis [23, 26]. The consequence of using these approximations on the extracted values of the astrophysical S-factors for the ${ }^{7} \mathrm{Be}(\mathrm{p}, \gamma)$ reaction in the sun is not easy to access. It might be necessary to review the results of some of these data, using a proper treatment of the relativistic corrections in the theory calculations used in the experimental analysis. Other improvements of the present formalism needs to be assessed. The relativistic effects in the nuclear interaction has to be studied in more depth. The effect of close Coulomb fields [27, 28] should also be considered in the case of dissociation of halo nuclei.

\begin{tabular}{|l|l|l|l|}
\hline Lab energy & $\Delta$ & $\Delta$ & $\Delta$ \\
{$[\mathrm{MeV} /$ nucleon $]$} & $E=0.1 \mathrm{MeV}$ & $E=1 \mathrm{MeV}$ & $E=2 \mathrm{MeV}$ \\
\hline 50 & $1.5 \%$ & $4.2 \%$ & $3.4 \%$ \\
\hline 80 & $3 \%$ & $5.5 \%$ & $4.1 \%$ \\
\hline 250 & $5.3 \%$ & $14.6 \%$ & $6.9 \%$ \\
\hline
\end{tabular}

Table 1: Relativistic corrections in the dissociation of ${ }^{8} \mathrm{~B}$ projectiles impinging on lead targets at different bombarding energies. $E$ is the relative energy of the proton and ${ }^{7} \mathrm{Be}$.

I would like to thank T. Aumann, H. Esbensen, K. Suemmerer and I. Thompson for beneficial discussions. This work was supported by the U.S. Department of Energy under grant No. DE-FG02-04ER41338.
[1] G. Baur, C.A. Bertulani and H. Rebel, Nucl. Phys. A458, 188 (1986).

[2] C.E. Rolfs, W. Rodney, "Cauldrons in the Cosmos", Chicago Press, 1988.

[3] C.A.Bertulani and L.F.Canto, Nucl. Phys. A540, 328 (1992).

[4] G. Baur, C.A. Bertulani and D.M. Kalassa, Nucl. Phys. A550, 527 (1992).

[5] K. Ieki et al., Phys. Rev. Lett. 70, 730 (1993).

[6] G.F. Bertsch and C.A. Bertulani, Nucl. Phys. A556, 136 (1993); C.A. Bertulani and G.F. Bertsch, Phys. Rev. C49, 2839 (1994); H. Esbensen, G.F. Bertsch and C.A. Bertulani, Nucl. Phys. A581, 107 (1995).

[7] G.H. Rawitscher, Phys. Rev. C9, 2210 (1974).

[8] Y. Sakuragi, M. Yashiro and M. Kamimura, Prog. Theor. Phys. Suppl. 89, 136 (1986).

[9] F.M. Nunes and I.J. Thompson, Phys. Rev. C57, R2818 (1998).

[10] F.M. Nunes and I.J. Thompson, Phys. Rev. C59, 2652 (1999).

[11] L.G. Arnold and B.C. Clark, Phys. Lett. B84, 46 (1979).

[12] J.S. Al-Khalili, J.A.Tostevin and J.M. Brooke, Phys. Rev. C55, R1018 (1997).

[13] H. Esbensen and G.F. Bertsch, Phys. Rev. C59, 3240 (1999).
[14] C.A. Bertulani, C.M. Campbell, and T. Glasmacher, Comput. Phys. Commun. 152, 317 (2003).

[15] H. De Vries, C.W. De Jager and C. De Vries, At. Data. Nucl. Data Tables 36, 495 (1987).

[16] G. Bertsch, J. Borysowicz, H. McManus, and W.G. Love, Nucl. Phys. A284, 399 (1977).

[17] L. Ray, Phys. Rev. C 20, 1857 (1979).

[18] H. Sagawa, private communication.

[19] H. Esbensen and G.F. Bertsch, Nucl. Phys. A706, 383 (2002).

[20] R.G.H. Robertson, Phys. Rev. C7, 543 (1973).

[21] H. Esbensen and G.F. Bertsch, Nucl. Phys. A600, 600 (1996).

[22] C.A. Bertulani, Z. Phys. A356, 293 (1996).

[23] B. Davids, et al., Phys. Rev. C63, 065806 (2001).

[24] T. Kikuchi et al., Phys. Lett. B391, 261 (1997).

[25] C.A. Bertulani and M. Gai, Nucl. Phys. A636, 227 (1998).

[26] F. Schümann et al., Phys. Rev. Lett. 90, 232501 (2003).

[27] H. Esbensen and C.A. Bertulani, Phys. Rev. C65, 024605 (2002).

[28] H. Esbensen, G.F. Bertsch and K. Snover, private communication and to be published. 